\title{
Super-resolution in live cells
}

Two strategies improve structured illumination microscopy for use in live-cell super-resolution imaging.

Super-resolution microscopy has provided groundbreaking views of biological structures. Yet issues such as phototoxicity and slow imaging speeds still practically limit most experiments to fixed cells or slow processes. Although these studies are informative, following the dynamics of subcellular structures at close to their true size and in real time remains an important unmet goal.

Eric Betzig, Dong Li (postdoc in Betzig's laboratory at Janelia Research Campus) and a team of researchers sought to improve live-cell super-resolution imaging of dynamic structures. They started with a method called structured illumination microscopy (SIM). Conventional,

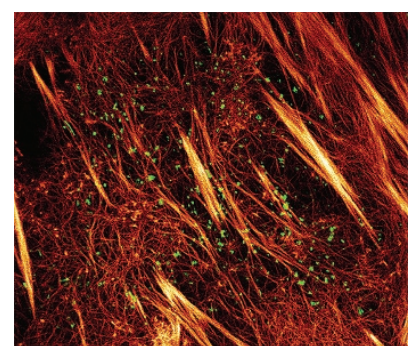

Actin (orange) and early endosomes (green) imaged with PA NL-SIM and TIRF-SIM, respectively. Reprinted from Li et al. (2015) with permission from AAAS. or linear, SIM has many advantages that make it useful for live-cell imaging, including rapid image acquisition at low doses of light. However, the principles that underlie SIM also limit it to approximately twofold improvements over diffraction-limited resolution.

Betzig was inspired by his late colleague Mats Gustafsson, who pioneered many SIM approaches, to extend the utility of SIM. He recalls "falling in love with SIM because of its much lower intensities and higher speeds and the beauty of the data [Gustafsson] could get on live cells." For this reason, his team sought to "find ways around the resolution limit without giving away more than we have to in terms of speed and intensity," he says.

They developed two approaches for enhancing resolution in live-cell SIM. The first was relatively straightforward; they carried out SIM on a total internal reflection fluorescence (TIRF) microscope with a high-numerical aperture (NA) objective (called high-NA TIRF-SIM).

\section{TARGETING SPINES}

A new probe allows for optical erasure of newly activated dendritic spines.

Optogenetic probes can be used to manipulate neuronal activity using light, but their targeting resolution is limited to entire neurons or bundles of projections. Uncaging techniques can target individual dendritic spines, but they cannot be used in vivo. Akiko Hayashi-Takagi of the University of Tokyo and colleagues have now described a probe for manipulating specific subsets of dendritic spines in vivo. This technique will allow researchers to causally link experience-dependent changes in spines with behavior, elucidating subcellular mechanisms of learning and allowing for manipulation of specific memories.

The probe is a modification of a photoactivatable form of the Rac1 small GTPase known as PaRac1, originally developed by Klaus Hahn (University of North Carolina, Chapel Hill) and colleagues. "I took advantage of PaRac1 because the prolonged activation of Rac1 could induce spine shrinkage," says Hayashi-Takagi. The authors fused PaRac1 to an inactive membrane protein that localizes to the post-synapse. They also added a targeting element from Arc mRNA, which leads to selective dendritic localization and translation of the mRNA in response to NMDA glutamate-receptor activation. In situ hybridization images that show the selective accumulation of Arc mRNA in the rodent hippocampus "are the most beautiful images I have ever seen in my research life," says Hayashi-Takagi.

The authors demonstrated the utility of their probe in hippocampal slice cultures, confirming that it was expressed specifically in spines activated by glutamate uncaging or by other means. Once induced, probe expression was maintained for about two days.

To the researchers, the real impetus to develop this probe was to look at the role of spines in behavior- "the biggest advantage of this probe," according to HayashiTakagi-which they did in mice trained in a motor task known as the rotarod. Learning 
Using this strategy, they were able to perform two-color, high-speed imaging at sub-100-nm resolution using a small fraction of the excitation light necessary for equivalent imaging with alternative approaches. However, the resolution was still limited.

To further improve the resolution achievable with SIM in live cells, the team developed a second approach, called patterned-activation nonlinear SIM (PA NL-SIM). Nonlinear SIM has been used to improve SIM resolution, but the increase in resolution is achieved by compromising imaging speed and light dosage, making it unsuitable for imaging processes in living cells.

PA NL-SIM makes use of photoswitchable fluorescent proteins. Prior to imaging, the fluorescent proteins are in a nonfluorescent state. Patterned light then switches a subset of them to the active state. This dynamic patterned activation means that every active molecule contributes useful photons to the image, allowing the researchers to achieve the same resolution as in conventional nonlinear SIM, but at higher speeds and with less light.

Developing PA NL-SIM was not without its challenges. One hurdle was finding an appropriate photoswitchable fluorescent protein. "You need a photoswitchable fluorescent protein that can take a licking and keep on ticking and undergo many switching cycles without bleaching," notes Betzig. He also explains that photoswitchable FPs in their off state must be completely off, as "glow in the inactive state can create background that hurts performance." He credits his collaborator Pingyong Xu for developing Skylan-NS, the protein that was used in their experiments to obtain super-resolution movies of structures such as the actin cytoskeleton with lateral resolution of $\sim 60 \mathrm{~nm}$. The team also combined PA NL-SIM with lattice light-sheet microscopy for 3D live-cell imaging of mitochondria and Golgi dynamics.

These new methods represent a major step toward the goal of imaging biologically relevant processes at super-resolution, and they stress the importance of imaging living cells with nontoxic doses of light. But Betzig notes that improvements in both optical systems and protein-labeling strategies will be important for future breakthroughs.

\section{Rita Strack}

\section{RESEARCH ARTICLES}

Li, D. et al. Extended-resolution structured illumination imaging of endocytic and cytoskeletal dynamics. Science 349, aab3500 (2015).

the rotarod induces the formation of new dendritic spines in motor cortex and requires Arc. The authors virally expressed the probe in the motor cortex and monitored spine dynamics with two-photon imaging through a cranial window, confirming that learning induced the formation of new spines.

One day after training, photoactivation of PaRac1 with an optical fiber through the cranial window led to selective shrinkage of newly formed spines. This optical erasure of newly formed spines in motor cortex was sufficient to block motor improvement due to training in subsequent behavioral tests. Retraining in the same task (after optical erasure of spines) led to repotentiation of the same ensemble of spines, suggesting that the spines involved in rotarod training were consistent over time.

Even more impressively, the authors were able to demonstrate the specificity of spine ensembles to a particular behavioral task. They sequentially trained mice on two different motor tasks, rotarod and beam, which led to the activation of two partially overlapping ensembles of spines. Optical erasure of spines potentiated in beam training (once PaRac1 expression in rotarod-potentiated spines had dissipated) selectively disrupted performance on the beam but not on the rotarod.

Dysfunctions in dendritic spines have been observed in numerous psychiatric disorders, but ascribing a causal role to spine changes in disease pathophysiology has been difficult. "No matter how well we would reveal the property of the dendritic spines in some psychiatric disease model mice ... these studies would not increase our understanding between the spine and disease pathophysiology," says Hayashi-Takagi. "That's why we launched the project to manipulate the spines systematically to challenge the causal relationship." The authors plan to use their probe to investigate the role of spine defects in post-traumatic stress disorder.

Brigitta B. Gundersen

RESEARCH PAPERS

Hayashi-Takagi, A. et al. Labelling and optical erasure of synaptic memory traces in the motor cortex. Nature 525, 333-338 (2015). 